\title{
Asymptotic Behavior of Solutions of Free Boundary Problem with Logistic Reaction Term
}

\author{
Jingjing Cai \\ School of Mathematics and Physics, Shanghai University of Electric Power, Pingliang Road 2103, Shanghai 200090, China \\ Correspondence should be addressed to Jingjing Cai; cjjing1983@163.com
}

Received 16 March 2014; Accepted 19 May 2014; Published 2 June 2014

Academic Editor: Junjie Wei

Copyright (C) 2014 Jingjing Cai. This is an open access article distributed under the Creative Commons Attribution License, which permits unrestricted use, distribution, and reproduction in any medium, provided the original work is properly cited.

We study a free boundary problem for a reaction diffusion equation modeling the spreading of a biological or chemical species. In this model, the free boundary represents the spreading front of the species. We discuss the asymptotic behavior of bounded solutions and obtain a trichotomy result: spreading (the free boundary tends to $+\infty$ and the solution converges to a stationary solution defined on $[0+\infty)$ ), transition (the free boundary stays in a bounded interval and the solution converges to a stationary solution with positive compact support), and vanishing (the free boundary converges to 0 and the solution tends to 0 within a finite time).

\section{Introduction}

Consider the following free boundary problem:

$$
\begin{gathered}
u_{t}=u_{x x}+u(1-u), \quad 0<x<h(t), t>0, \\
u(t, 0)=u(t, h(t))=0, \quad t>0, \\
h^{\prime}(t)=-\mu u_{x}(t, h(t))-\mu \alpha, \quad t>0, \\
h(0)=h_{0}, \quad u(0, x)=u_{0}(x), \quad 0 \leq x \leq h_{0},
\end{gathered}
$$

where $x=h(t)$ is a moving boundary to be determined together with $u(t, x)$ and $\alpha>0$ is a given constant. The initial function $u_{0}$ belongs to $\mathcal{Y}\left(h_{0}\right)$ for some $h_{0}>0$, where

$$
\begin{gathered}
\mathcal{Y}\left(h_{0}\right):=\left\{\phi \in C^{2}\left(\left[0, h_{0}\right]\right): \phi(0)=\phi\left(h_{0}\right)=0,\right. \\
\left.\phi(x) \geq(\not \equiv) 0 \text { in }\left(0, h_{0}\right)\right\} .
\end{gathered}
$$

Recently, problem (1) with $\alpha=0$ was studied by [1-3] and so forth. They used this model to describe the spreading of a new or invasive species; they used the free boundary $h(t)$ which represents the expanding front of the species whose density is represented by $u(t, x)$. They obtained a spreadingvanishing dichotomy result; namely, the species either spreads to the whole environment and stabilizes at the positive state 1 (i.e., $u \rightarrow 1$ ) or vanishes (i.e., $u \rightarrow 0$ ) as time goes to infinity. Such a result shows that problem (1) with $\alpha=$ 0 has advantages comparing with the Cauchy problems (the Cauchy problems have hair-trigger effect: any positive solution which converges to a positive constant; cf. $[4,5])$. In the last two years, [6] also studied the corresponding problem of (1) with $\alpha=0$ in high dimension spaces.

In this paper, we mainly study problem (1) with $\alpha>0$; such a boundary condition represents that there is a spreading resistant force at the front for some species. Intuitively, the presence of $\alpha>0$ makes the solution more difficult to spread than the case where $\alpha=0$. Indeed, $h^{\prime}(t)>0$ only if $u_{x}(t, h(t))<-\alpha$. This boundary condition is widely used in many biological models. For example, it is often used in protocell models (cf. $[7,8]$ ).

We give the following theorem whose proof is similar to that of $[1,2]$. It suffices to repeat their arguments with obvious modification.

Theorem 1. For any given $\gamma \in(0,1)$, there is a $T \in(0,+\infty)$ such that free boundary problem (1) has a solution

$$
(u, h) \in C^{((1+\gamma) / 2), 1+\gamma}\left(\bar{D}_{T}\right) \times C^{1+\gamma / 2}([0, T]),
$$


where $D_{T}:=\left\{(t, x) \in \mathbb{R}^{2}: x \in[0, h(t)], t \in(0, T]\right\}$, and the solution can be extended to some interval $\left(0, T_{0}\right)$ with $T_{0}>T$ as long as $\inf _{0<t<T} h(t)>0$.

Moreover, as in the proof of [9, Lemma 2.8], one can show that $h_{\infty}:=\lim _{t \rightarrow T} h(t) \in[0,+\infty]$ exist.

The main purpose of this paper is to study the asymptotic behavior of bounded solutions of (1) and obtain trichotomy result. We will prove that, for a solution $(u, h)$ of $(1)$, one has either

(i) spreading: $h_{\infty}=+\infty$ and

$$
\lim _{t \rightarrow \infty} u(t, x)=w(x) \quad \text { locally uniformly in }(0,+\infty),
$$

where $w$ is the unique positive solution of

$$
\begin{aligned}
q^{\prime \prime}+q(1-q) & =0, \quad x>0, \\
q(0) & =0,
\end{aligned}
$$

or

(ii) vanishing: $\lim _{t \rightarrow T} h(t)=0$ and

$$
T<+\infty, \quad \lim _{t \rightarrow T 0 \leq x \leq h(t)} \max _{t} u(t, x)=0
$$

or

(iii) transition: $0<h_{\infty}<+\infty$ and

$$
\lim _{t \rightarrow \infty} u(t, \cdot)=v(\cdot) \quad \text { locally uniformly in }\left(0, h_{\infty}\right),
$$

where $v$ is the solution of

$$
\begin{aligned}
& v^{\prime \prime}+v(1-v)=0, \quad x \in\left(0, h_{\infty}\right), \\
& v(0)=v\left(h_{\infty}\right)=0, \quad-v^{\prime}\left(h_{\infty}\right)=\alpha .
\end{aligned}
$$

Remark 2. Comparing with the results in [1-3], the phenomenon (iii) is a new one, since it does not happen in case $\alpha=$ 0 .

Remark 3. (ii) shows that vanishing happens in a finite time and the free boundary converges to the point 0 ; those phenomena are also new and do not happen in case $\alpha=0$.

\section{Asymptotic Behavior of Solutions}

In this section, we study the asymptotic behavior of solutions and obtain trichotomy result when $\alpha<\sqrt{3} / 3$; namely, the solution of (1) is either vanishing (Theorem 6) or transition (Theorem 7) or spreading (Theorem 10). Then, we prove that only vanishing happens if $\alpha \geq \sqrt{3} / 3$ (Theorem 11) for the completeness of the paper.

We first prepare the following comparison theorems which can be proved similarly as in [2, Lemma 3.5].
Lemma 4. Suppose that $T \in(0, \infty), \bar{h} \in C^{1}([0, T])$, and $\bar{u} \epsilon$ $C\left(\bar{D}_{T}\right) \cap C^{1,2}\left(D_{T}\right)$ with $D_{T}=\left\{(t, x) \in \mathbb{R}^{2}: 0<t \leq T, 0<x<\right.$ $\bar{h}(t)\}$ and

$$
\begin{gathered}
\bar{u}_{t} \geq \bar{u}_{x x}+\bar{u}(1-\bar{u}), \quad 0<t \leq T, \quad 0<x<\bar{h}(t), \\
\bar{u}(t, 0) \geq 0, \quad \bar{u}(t, \bar{h}(t))=0, \quad 0<t \leq T, \\
\bar{h}^{\prime}(t) \geq-\mu \bar{u}(t, \bar{h}(t))-\mu \alpha, \quad 0<t \leq T .
\end{gathered}
$$

If $h_{0} \leq \bar{h}(0)$ and $u_{0}(x) \leq \bar{u}(0, x)$ in $\left[0, h_{0}\right]$ and if $(u, h)$ is a solution of (1), then

$$
\begin{array}{r}
h(t) \leq \bar{h}(t), \quad u(x, t) \leq \bar{u}(x, t) \quad \text { for } t \in(0, T], \\
x \in(0, h(t)) .
\end{array}
$$

Remark 5. The pair $(\bar{u}, \bar{h})$ is usually called an upper solution of problem (1) and one can define a lower solution by revising all the inequalities.

Theorem 6. Let $(u, h)$ be a solution of (1) on $\left[0, T^{*}\right)$. If $\lim _{t \rightarrow T^{*}} h(t)=0$, then $T^{*}<+\infty$ and

$$
\lim _{t \rightarrow T^{*} 0 \leq x \leq h(t)} \max _{0} u(t, x)=0 .
$$

Proof. By $[2,10]$, one can prove that there exists a constant $C_{1}$ such that $u(t, x) \leq C_{1}$. In order to prove that $u$ converges to 0 , we need to construct the function

$$
U(t, x):=C_{1}\left[2 M(h(t)-x)-M^{2}(h(t)-x)^{2}\right]
$$

over the region

$$
Q:=\left\{(t, x): 0<t<T^{*}, \max \left\{h(t)-M^{-1}, 0\right\}<x<h(t)\right\},
$$

where

$$
M:=\max \left\{\frac{\alpha+\sqrt{\alpha^{2}+2}}{2}, \frac{4\left\|u_{0}\right\|_{C^{1}\left(\left[-h_{0}, h_{0}\right]\right)}}{3 C_{1}}\right\} .
$$

Clearly $0 \leq U \leq C_{1}$ in $Q$. By the definitions of $U$ and $M$, we have

$$
U_{t}-U_{x x}-U(1-U) \geq C_{1}\left(2 M^{2}-2 M \alpha-1\right) \geq 0 \quad \text { in } Q .
$$

Moreover,

$$
\begin{gathered}
U(t, h(t))=u(t, h(t))=0 \quad \text { for } t \in\left(0, T^{*}\right), \\
U(t, 0)>0=u(t, 0) \quad \text { when } h(t)<M^{-1} .
\end{gathered}
$$

Therefore, $u(t, x) \leq U(t, x)$ in $Q$ by the comparison principle Lemma 4. Note that $\lim _{t \rightarrow T^{*}} h(t)=0$; then there exists $T_{1}<$ $T^{*}$ such that $h(t)-M^{-1}<0$ for $t>T_{1}$. Therefore, $u(t, x) \leq$ $U(t, x)$ for $t>T_{1}$ and $x \in[0, h(t)]$. For such $t$ and $x$, we have

$$
U(t, x) \leq 2 M C_{1} h(t) \longrightarrow 0 \quad \text { as } t \longrightarrow T^{*} ;
$$


it follows that

$$
\|u(t, \cdot)\|_{L^{\infty}([0, h(t)])} \longrightarrow 0 \quad \text { as } t \longrightarrow T^{*} .
$$

We now prove that $T^{*}<+\infty$. By $\lim _{t \rightarrow T^{*}} h(t)=0$, there is some $L_{*}>0$ such that

$$
h(t) \leq L_{*} \quad \text { for } t \in\left[0, T^{*}\right) .
$$

Set $L:=2\left(1+L_{*}\right)$ and

$$
\xi_{0}(x):=\frac{2 \varepsilon}{L^{2}}\left(L^{2}-x^{2}\right),
$$

where $\varepsilon>0$ is small such that

$$
8\left(\alpha+\sqrt{\alpha^{2}+2}\right) \varepsilon \leq \alpha, \quad 32 \varepsilon \leq \alpha .
$$

Consider the problem

$$
\begin{gathered}
\xi_{t}=\xi_{x x}+2 \xi\left(1-\frac{\xi}{2 \varepsilon}\right), \quad 0<x<\bar{h}(t), \quad t>0, \\
\xi(t, 0)=\xi(t, \bar{h}(t))=0, \quad t>0, \\
\bar{h}^{\prime}(t)=-\mu \xi_{x}(t, \bar{h}(t))-\mu \alpha, \quad t>0, \\
\bar{h}(0)=L, \quad \xi(0, x)=\xi_{0}(x), \quad 0 \leq x \leq L .
\end{gathered}
$$

It is obvious that $\xi(t, x) \leq 2 \varepsilon$ for all $t \geq 0$. Construct a function

$$
U^{\varepsilon}(t, x):=2 \varepsilon\left[2 M(\bar{h}(t)-x)-M^{2}(\bar{h}(t)-x)^{2}\right]
$$

over $\bar{Q}:=\left\{(t, x): t>0, \max \left\{0, \bar{h}(t)-M^{-1}\right\} \leq x \leq \bar{h}(t)\right\}$, where $M:=\max \left\{\alpha+\sqrt{\alpha^{2}+2}, 4\right\}$. Then $U^{\varepsilon}(t, x)$ is an upper solution of (22) over $\bar{Q}$ and so

$$
-\xi_{x}(t, \bar{h}(t)) \leq-U_{x}^{\varepsilon}(t, \bar{h}(t))=4 M \varepsilon \leq \frac{\alpha}{2} .
$$

Therefore, $\bar{h}^{\prime}(t) \leq-\alpha \mu / 2$. Thus, $\bar{h}(t) \rightarrow 0$ as $t \rightarrow \bar{T}^{*} \leq$ $2 L / \alpha \mu$.

On the other hand, (18) implies that there exists some $T_{0} \epsilon$ $\left(0, T^{*}\right)$ such that $u(t, x) \leq \varepsilon$ for all $x \in[0, h(t)]$ and $t>T_{0}$. Clearly $\xi_{0}(x) \geq u\left(T_{0}, x\right)$ for $x \in\left[0, h\left(T_{0}\right)\right]$. By the comparison principle, we have $h\left(t+T_{0}\right) \leq \bar{h}(t)$, and so $T^{*}$ cannot be $\infty$.

Theorem 7. Assume that $0<\alpha<\sqrt{3} / 3$. Let $(u, h)$ be a solution of (1). If $0<h_{\infty}<+\infty$, then

$$
\begin{aligned}
& h_{\infty}=L_{\alpha}, \\
& \quad \lim _{t \rightarrow \infty} u(t, \cdot)=v_{\alpha}(\cdot) \quad \text { locally uniformly in }\left(0, h_{\infty}\right),
\end{aligned}
$$

where $v_{\alpha}$ is a unique positive solution of

$$
\begin{gathered}
v^{\prime \prime}+v(1-v)=0, \quad 0<x<L_{\alpha}, \\
v(0)=v\left(L_{\alpha}\right)=0, \quad v^{\prime}(0)=-v^{\prime}\left(L_{\alpha}\right)=\alpha,
\end{gathered}
$$

where

$$
L_{\alpha}:=2 \int_{0}^{B} \frac{d r}{\sqrt{\alpha^{2}-r^{2}+(2 / 3) r^{3}}}
$$

with $B \in(0,1)$ given by $\alpha^{2}=2 \int_{0}^{B} s(1-s) d s$.

Remark 8. This is a new phenomenon. It never happens when $\alpha=0$. Moreover, by the phase plane method, one can prove that $v_{\alpha} \rightarrow 0$ and $L_{\alpha} \rightarrow \pi$ as $\alpha \rightarrow 0$. This conclusion gives an explanation of Lemma 3.1 in [2]; that is, vanishing happens if $h_{\infty} \leq \pi$.

Remark 9. It is easily seen that (26) has no positive solution when $\alpha \geq 2 \int_{0}^{1} s(1-s) d s=\sqrt{3} / 3$.

Proof of Theorem 7. For any $\varepsilon>0$, there exists $t^{*}>0$ such that $h_{\infty}-\varepsilon<h(t)<h_{\infty}+\varepsilon$ for $t>t^{*}$. Let $\bar{u}_{0}(x)$ be a function defined on $\left(0, h_{\infty}+\varepsilon\right)$ and satisfies

$$
\begin{gathered}
\bar{u}_{0}(x) \geq u\left(t^{*}, x\right) \quad \text { for } x \in\left(0, h_{\infty}\right), \\
\bar{u}_{0}(0)=\bar{u}_{0}\left(h_{\infty}+\varepsilon\right)=0 .
\end{gathered}
$$

By the comparison principle we have $u(t, x) \leq \bar{u}(t, x)$ in $\left(t^{*}, \infty\right) \times(0, h(t))$, where $\bar{u}(t, x)$ is the solution of

$$
\begin{gathered}
\bar{u}_{t}=\bar{u}_{x x}+\bar{u}(1-\bar{u}), \quad t>t^{*}, 0<x<h_{\infty}+\varepsilon, \\
\bar{u}(t, 0)=\bar{u}\left(t, h_{\infty}+\varepsilon\right)=0, \quad t>t^{*}, \\
\bar{u}\left(t^{*}, x\right)=\bar{u}_{0}(x), \quad 0<x<h_{\infty}+\varepsilon .
\end{gathered}
$$

It is well known that

$$
\begin{aligned}
& \text { (i) } \bar{u} \rightarrow 0 \text { as } t \rightarrow \infty \text { if } h_{\infty}+\varepsilon \leq \pi \text {; or } \\
& \text { (ii) } \bar{u} \rightarrow \bar{u}_{\varepsilon}^{*} \text { as } t \rightarrow \infty \text { if } h_{\infty}+\varepsilon>\pi \text {, }
\end{aligned}
$$

where $\bar{u}_{\varepsilon}^{*}$ is a positive function. More precisely, when $h_{\infty}+\varepsilon>$ $\pi$, it follows from [11, Corollary 3.4] that $\bar{u}_{\varepsilon}^{*}$ is the unique positive solution of

$$
\begin{gathered}
\left(\bar{u}_{\varepsilon}^{*}\right)^{\prime \prime}+\bar{u}_{\varepsilon}^{*}\left(1-\bar{u}_{\varepsilon}^{*}\right)=0, \quad 0<x<h_{\infty}+\varepsilon, \\
\bar{u}_{\varepsilon}^{*}\left(h_{\infty}+\varepsilon\right)=\bar{u}_{\varepsilon}^{*}(0)=0 .
\end{gathered}
$$

Hence,

$$
\lim _{t \rightarrow \infty} u(t, x)=0, \quad \text { or } \limsup _{t \rightarrow \infty} u(t, x) \leq \bar{u}_{\varepsilon}^{*} .
$$

Similarly,

$$
\liminf _{t \rightarrow \infty} u(t, x) \geq \underline{u}_{\varepsilon}^{*}(x) \quad \text { when } h_{\infty}-\varepsilon>\pi,
$$

where $\underline{u}_{\varepsilon}^{*}(x)$ is a positive solution of

$$
\begin{gathered}
\left(\underline{u}_{\varepsilon}^{*}\right)^{\prime \prime}+\underline{u}_{\varepsilon}^{*}\left(1-\underline{u}_{\varepsilon}^{*}\right)=0, \quad 0<x<h_{\infty}-\varepsilon, \\
\underline{u}_{\varepsilon}^{*}\left(h_{\infty}-\varepsilon\right)=\underline{u}_{\varepsilon}^{*}(0)=0 .
\end{gathered}
$$

We conclude from (31) and (32) that

$$
\lim _{t \rightarrow \infty} u(t, x)=0 \quad \text { if } h_{\infty} \leq \pi,
$$


or when $h_{\infty}>\pi$,

$$
\lim _{t \rightarrow \infty} u(t, x)=u^{*}(x) \quad \text { locally uniformly in }\left(0, h_{\infty}\right) \text {, }
$$

where $u^{*}(x)$ is the unique positive solution of

$$
\begin{gathered}
\left(u^{*}\right)^{\prime \prime}+u^{*}\left(1-u^{*}\right)=0, \quad 0<x<h_{\infty}, \\
u^{*}\left(h_{\infty}\right)=u^{*}(0)=0 .
\end{gathered}
$$

We now show that $\lim _{t \rightarrow \infty} u(t, x)=0$ is impossible when $h_{\infty}>0$. Suppose that this does not hold; there exists $L_{0}$ such that $h(t) \leq L_{0}$. Then using the approach of proving $T^{*}<+\infty$ in Theorem 7, we can show that $\lim _{t \rightarrow T} h(t)=0$ for some $0<$ $T<+\infty$; this contradicts the assumption $h_{\infty}>0$. Hence, $\lim _{t \rightarrow \infty} u(t, x)=u^{*}(x)$, locally uniformly in $\left(0, h_{\infty}\right)$; we next prove that $u^{*}(x)=v_{\alpha}(x)$.

Make a change of the variable $x$ to reduce $[0, h(t)]$ to the fixed interval $\left[0, h_{0}\right]$ and use $L^{p}$ estimates as well as Sobolev embedding theorems on the reduced equation with Dirichlet boundary conditions to conclude that

$$
\left\|u(t, \cdot)-u^{*}(\cdot)\right\|_{C^{1+(\gamma / 2)}}([0, h(t)]) \longrightarrow 0 \quad(t \longrightarrow \infty)
$$

for some $\gamma>0$. It follows that $h^{\prime}(t)=-\mu u_{x}(t, h(t))-\mu \alpha \rightarrow$ $-\mu\left(u^{*}\right)^{\prime}\left(h_{\infty}\right)-\mu \alpha$ as $t \rightarrow \infty$. Hence, we conclude that $\left(0, h_{\infty}\right)$ is not a finite interval unless $-\left(u^{*}\right)^{\prime}\left(h_{\infty}\right)=\alpha$.

Theorem 10. Let $(u, h)$ be a solution of $(1)$. If $h_{\infty}=+\infty$, then

$$
\lim _{t \rightarrow \infty} u(t, x)=w(x) \quad \text { locally uniformly in }[0,+\infty) \text {, }
$$

where $w$ is the unique positive solution of

$$
\begin{gathered}
q^{\prime \prime}+q(1-q)=0, \quad x>0, \\
q(0)=0 .
\end{gathered}
$$

Proof. Choose a bounded continuous function $W_{0}(x) \geq$ $u_{0}(x)$ for $x \in\left[0, h_{0}\right]$ and $W_{0} \geq 0$ for $x \in[0,+\infty)$. Let $W(t, x)$ be the unique solution of

$$
\begin{gathered}
W_{t}=W_{x x}+W(1-W), \quad t>0, x>0, \\
W(t, 0)=0, \quad t>0, \\
W(0, x)=W_{0}(x), \quad x>0 .
\end{gathered}
$$

Then the comparison principle theorem shows that $u(t, x) \leq$ $W(t, x)$ for $t>0, x>0$. Using [11, Lemma 3.4], we see that

$$
\limsup _{t \rightarrow \infty} u(t, x) \leq \lim _{t \rightarrow \infty} W(t, x)=w(x) \quad \text { for } x \in[0,+\infty) .
$$

On the other hand, since $h_{\infty}=+\infty$, for any large $l>\pi$, there is $\tau>0$ such that $h(\tau)=l$ and $h(t) \geq l$ for all $t>\tau$. Let $\underline{u}_{l}(t, x)$ be the solution of the following problem:

$$
\begin{gathered}
\underline{u}_{t}=\underline{u}_{x x}+\underline{u}(1-\underline{u}), \quad t>\tau, 0<x<l, \\
\underline{u}(t, 0)=\underline{u}(t, l)=0, \quad t>\tau, \\
\underline{u}(0, x)=\psi(x), \quad 0<x<l,
\end{gathered}
$$

where $\psi$ is a nonnegative continuous function satisfying $\psi(x) \leq u(\tau, x)$ for $0<x<l$. The comparison principle implies

$$
\underline{u}_{l}(t, x) \leq u(t, x) \text { for } t>\tau, 0 \leq x \leq l \text {. }
$$

By [11], one can obtain

$$
\lim _{t \rightarrow \infty} \underline{u}_{l}(t, x)=v_{l}(x) \quad \text { uniformly in }[0, l]
$$

where $v_{l}$ is the positive solution of

$$
\begin{gathered}
v^{\prime \prime}+v(1-v)=0, \quad 0<x<l, \\
v(0)=v(l)=0,
\end{gathered}
$$

It is well known that $\lim _{l \rightarrow \infty} v_{l}(x)=w(x)$. Combining this with (43) and (44), we have

$$
w(x) \leq \liminf _{t \rightarrow \infty} u(t, x)
$$

By (41) and (46), we have

$$
\lim _{t \rightarrow \infty} u(t, x)=w(x)
$$

Theorem 11. Suppose that $\alpha \geq \sqrt{3} / 3$ and $(u, h)$ is a solution of (1) defined on some maximal existence interval $\left[0, T_{*}\right)$; then $T_{*}<+\infty$, $u$ converges to 0 as $t \rightarrow T_{*}$, and $\lim _{t \rightarrow T_{*}} h(t)=0$.

Proof. The proof of this theorem is similar to [10]; it suffices to repeat their arguments with obvious modification.

\section{Example}

In this section, we give some sufficient conditions for vanishing, spreading, and transition.

Example 1. Suppose that $\alpha<\sqrt{3} / 3$. Let $h_{0}>0$ and $u_{0}(x) \epsilon$ $\mathscr{Y}\left(h_{0}\right)$; then the following properties hold:

(i) vanishing happens when $u_{0}(x)<v_{\alpha}(x)$;

(ii) spreading happens if $u_{0}(x)>v_{\alpha}(x)$ for $x \in\left[0, h_{0}\right]$;

(iii) transition happens if $u_{0}(x) \equiv v_{\alpha}(x)$ for $x \in\left[0, h_{0}\right]$.

Proof. (i) By [1], we see that $v_{\alpha_{1}}(x)<v_{\alpha_{2}}(x)$ for $\alpha_{1}<\alpha_{2}$. Since $u_{0}(x)<v_{\alpha}(x)$, there is $\beta<\alpha$ such that $u_{0}(x)<v_{\beta}(x)$, by the comparison principle that $u(t, x)<v_{\beta}(x)$, so $h_{\infty} \neq+\infty$ and $h_{\infty} \neq L_{\alpha}$. It then follows from Theorem 6 that vanishing happens.

(ii) Let $(u, h)$ be a solution of (1) with initial data $u_{0}(x)$; by the phase plane analysis, there is $\gamma>\alpha$ such that $u_{0}(x)>$ $v_{\gamma}(x)$. It then follows from the comparison principle that $u(t, x)>v_{\gamma}(x)$, so Theorem 10 implies that $h_{\infty}=+\infty$ and spreading happens.

(iii) It follows from the comparison principle Lemma 4 that $u(t, x) \equiv v_{\alpha}(x)$ and $h(t) \equiv L_{\alpha}$ for all $t>0$. 


\section{Conflict of Interests}

The author declares that there is no conflict of interests regarding the publication of this paper.

\section{Acknowledgments}

This research was supported by Shanghai Natural Science Foundation (no. 13ZR1454900) and Shanghai University Young Teachers Training Scheme (no. ZZsdl13021).

\section{References}

[1] Y. Du and B. Lou, "Spreading and vanishing in nonlinear diffusion problems with free boundaries," http://arxiv.org/abs/ 1301.5373.

[2] Y. Du and Z. Lin, "Spreading-vanishing dichotomy in the diffusive logistic model with a free boundary," SIAM Journal on Mathematical Analysis, vol. 42, no. 1, pp. 377-405, 2010.

[3] Y. Kaneko and Y. Yamada, "A free boundary problem for a reaction-diffusion equation appearing in ecology," Advances in Mathematical Sciences and Applications, vol. 21, no. 2, pp. 467492, 2011.

[4] D. G. Aronson and H. F. Weinberger, "Multidimensional nonlinear diffusion arising in population genetics," Advances in Mathematics, vol. 30, no. 1, pp. 33-76, 1978.

[5] D. G. Aronson and H. F. Weinberger, "Nonlinear diffusion in population genetics, combustion, and nerve pulse propagation," in Partial Differential Equations and Related Topics, vol. 446 of Lecture Notes in Mathematics, pp. 5-49, Springer, Berlin, Germany, 1975.

[6] Y. Du and Z. Guo, "The Stefan problem for the Fisher-KPP equation," Journal of Differential Equations, vol. 253, no. 3, pp. 996-1035, 2012.

[7] A. Friedman and B. Hu, "A Stefan problem for a photocell model," SIAM Journal on Mathematical Analysis, vol. 30, no. 4, pp. 912-926, 1999.

[8] H. Zhang, C. Qu, and B. Hu, "Bifurcation for a free boundary problem modeling a protocell," Nonlinear Analysis: Theory, Methods \& Applications, vol. 70, no. 7, pp. 2779-2795, 2009.

[9] J. Cai, B. Lou, and M. Zhou, "Asymptotic behavior of solutions of a reaction diffusion equation with free boundary conditions," Preprint.

[10] J. Cai, "Asymptotic behavior of solutions of Fisher-KPP equation with free boundary conditions," Nonlinear Analysis: Real World Applications, vol. 16, pp. 170-177, 2014.

[11] R. S. Cantrell and C. Cosner, Spatial Ecology via ReactionDiffusion Equations, John Wiley \& Sons, Chichester, UK, 2003. 


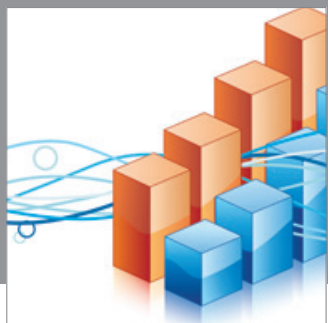

Advances in

Operations Research

mansans

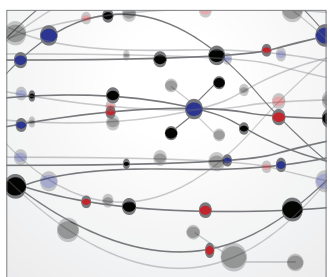

The Scientific World Journal
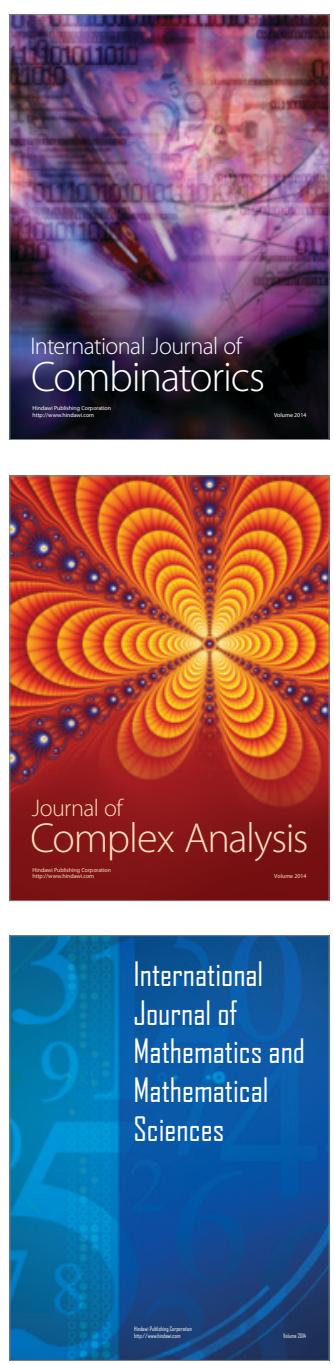
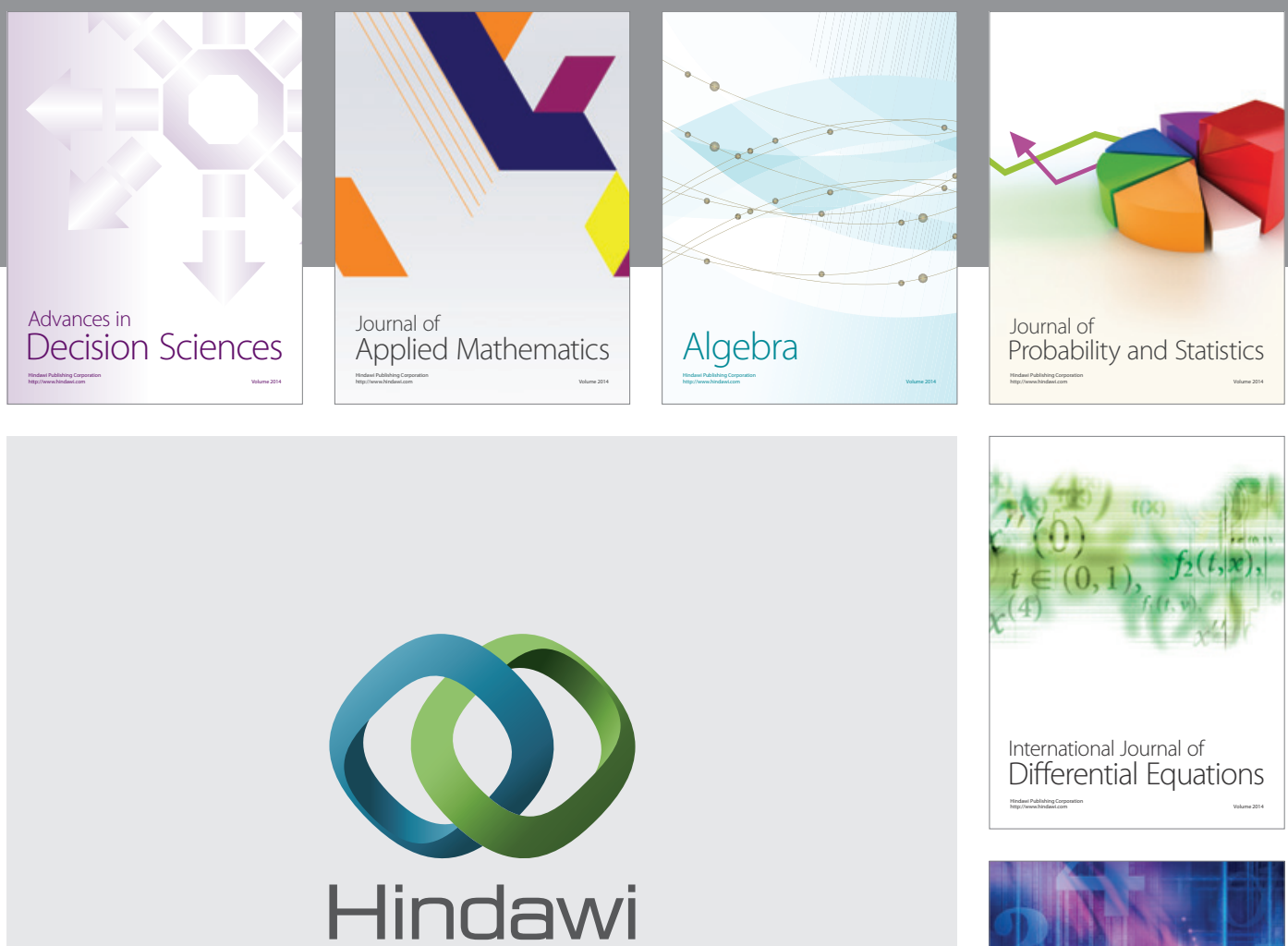

Submit your manuscripts at http://www.hindawi.com
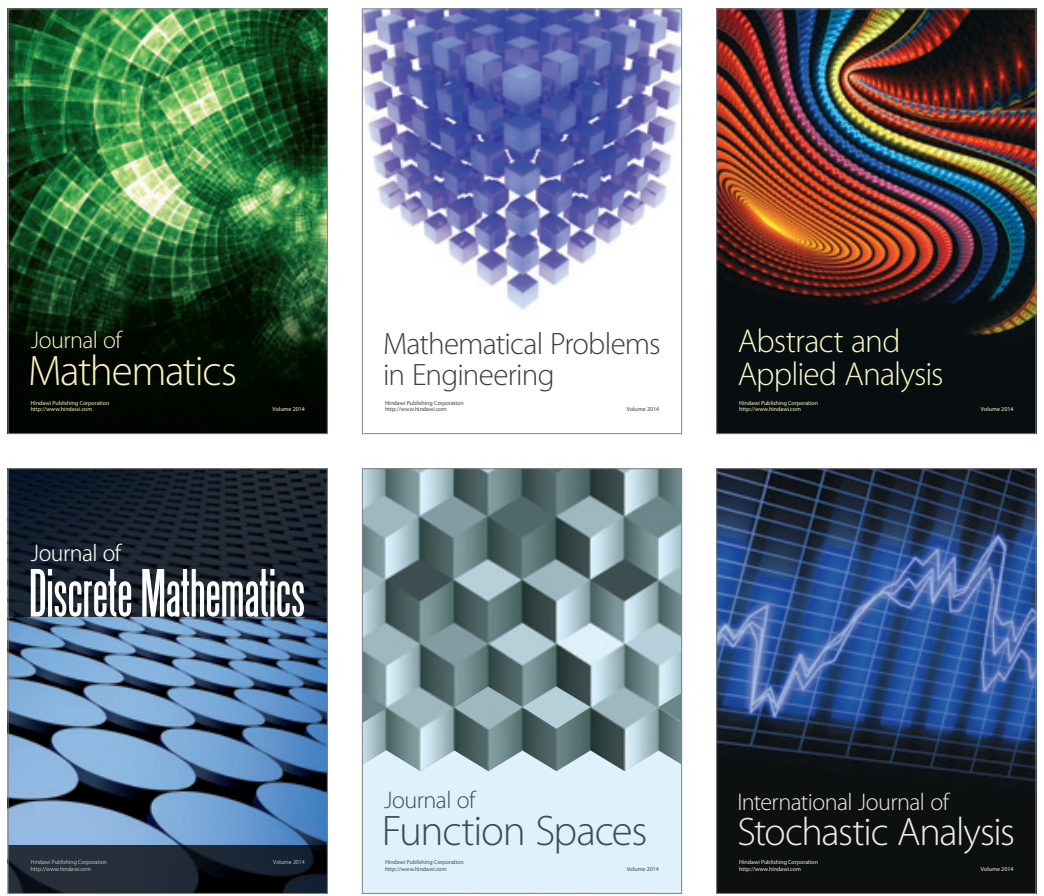

Journal of

Function Spaces

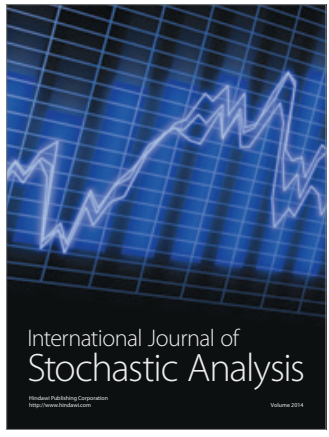

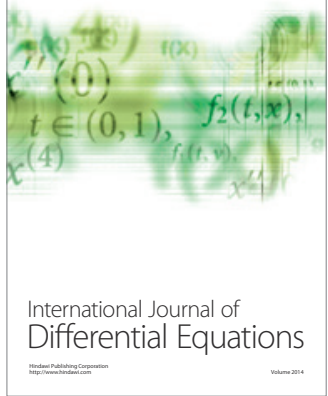
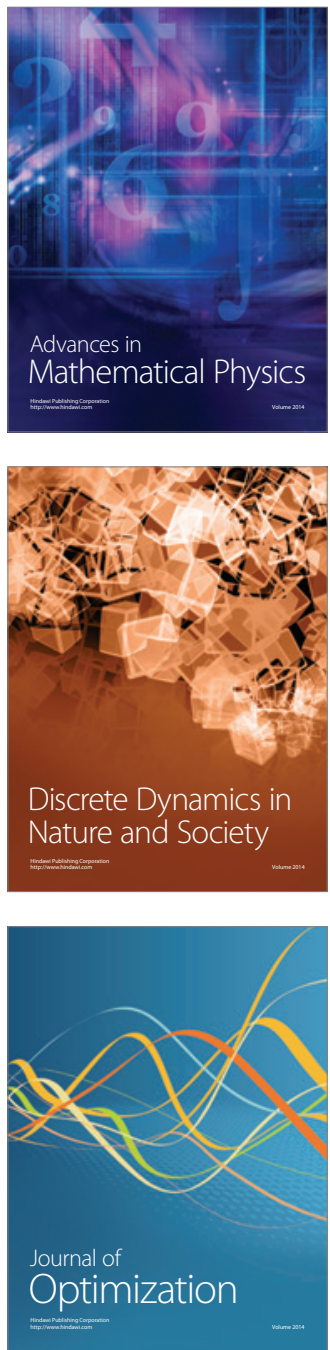